\title{
Histone preconditioning protects against obstructive jaundice-induced liver injury in rats
}

\author{
YOU-XING ZHOU ${ }^{1}$, YONG NI ${ }^{1}$, YI-BING LIU ${ }^{2}$ and XIAOHONG LIU ${ }^{3}$ \\ ${ }^{1}$ Department of Hepatobiliary Surgery, The First Affiliated Hospital of Shenzhen University, Shenzhen, Guangdong 518035; \\ ${ }^{2}$ Department of Hepatobiliary Surgery, Longgang District Central Hospital of Shenzhen, Shenzhen, Guangdong 518116; \\ ${ }^{3}$ Department of Geriatric Psychiatry, Wuxi Mental Health Center, Wuxi, Jiangsu 214151, P.R. China
}

Received November 25, 2013; Accepted March 25, 2014

DOI: $10.3892 /$ etm.2014.1697

\begin{abstract}
A major consequence of obstructive jaundice (OJ) in clinical practice is the development of severe liver injury, and at present, no effective treatments have been developed to protect against it. Preconditioning with damage-associated molecular pattern (DAMP) molecules has been demonstrated to protect multiple organs from injury, and histones have been recently identified as DAMP molecules. The aim of the present study was to investigate the protective effect of histone preconditioning against OJ-induced liver injury in rats and the involvement of Toll-like receptors. Rats were administered histone proteins $(200 \mu \mathrm{g} / \mathrm{kg} ; 1 \mathrm{ml})$ or physiological saline (1 $\mathrm{ml}$ ) intraperitoneally $24 \mathrm{~h}$ prior to being subjected to bile duct ligation (BDL). The serum levels of liver enzymes and bilirubin, as well as the histopathology were analyzed. The mRNA expression of interleukin-6 (IL-6) in the liver tissue was analyzed using quantitative polymerase chain reaction. BDL in the control group caused severe OJ-induced liver injury, as indicated by the significantly elevated levels of liver enzymes and mRNA levels of IL-6, and confirmed by histopathological alterations. However, histone preconditioning significantly ameliorated the OJ-induced liver injury caused by BDL, as shown by an improvement in the levels of liver enzymes, a suppression of IL-6 production, as well as histopathological alterations. Therefore, these results suggested that histone preconditioning is able to protect against OJ-induced liver injury in rats.
\end{abstract}

\section{Introduction}

Obstructive jaundice (OJ) is a common pathophysiological process that occurs in numerous clinical conditions, including gallstones, stricture of the bile duct and pancreatic cancer.

Correspondence to: Professor Yong $\mathrm{Ni}$, Department of Hepatobiliary Surgery, The First Affiliated Hospital of Shenzhen University, 3002 West Sungang Road, Shenzhen, Guangdong 518035, P.R. China

E-mail: daolangxin@126.com

Key words: histone, precondition, obstructive jaundice, liver injury
Surgical or interventional decompression is the main treatment strategy for OJ patients (1). In the treatment of OJ patients, severe complications may occur in surgical or interventional decompression. The procedure of decompression itself is not enough to prevent the complications. One of the major consequences of $\mathrm{OJ}$ is the development of severe liver injury (2). The mechanisms responsible for the pathogenesis of OJ-induced liver injury remain largely unknown, although inflammatory cell infiltration, microvascular perfusion failure and Toll-like receptor (TLR) activation are reported to be involved $(3,4)$. At present, there are no effective treatments to protect against OJ-induced liver injury, thus, novel therapeutic strategies are urgently required.

Preconditioning is a process where the body is subjected to mild stress in order to increase its resistance to further stresses. It has been associated with increased resistance and protection against numerous types of tissue injuries, including infected thermal injury, ischemia/reperfusion (I/R) injury and hemorrhagic shock (5-8). Notably, hyperthermia preconditioning has been demonstrated to be an effective method to protect against OJ-induced liver injury in rats (9). The results from this study were in accordance with previous studies, which demonstrated that hyperthermic preconditioning enhances the immune response of rats with OJ $(10,11)$.

Previous studies have demonstrated that numerous damage-associated molecular pattern (DAMP) molecules, including high mobility group box 1 (HMGB1), lipoteichoic acid, lipopolysaccharide (LPS) and heat shock protein, have been successfully developed as preconditioning agents (12-15). Furthermore, the protective effect of HMGB1 preconditioning on hepatic I/R injury was found to involve the downregulation of it's receptor TLR4 (16). It is noteworthy that pretreatment with LPS, lipoteichoic acid and HMGB1 were all demonstrated to have a protective effect against myocardial I/R injury, suggesting that cross-tolerization may occur between different preconditioning agents (12-14). Histones have been previously identified as alarmins or DAMP molecules, which serve as danger signals in the context of the 'danger model' to promote activation of the innate immune system in response to several types of tissue injury, including OJ-induced liver injury (17).

Therefore, in the present study it was hypothesized that preconditioning with histones, which are recently identified DAMP molecules, may protect against OJ-induced liver 
injury and the downregulation of TLR may be involved in this process.

\section{Materials and methods}

Reagents. Histones obtained from calf thymus (H9250) were purchased from Sigma-Aldrich (St. Louis, MO, USA). TRIzol reagent was purchased from Invitrogen Life Technologies (Carlsbad, CA, USA). The RevertAid First Strand cDNA Synthesis kit was obtained from Fermantas (Beverly, MA, USA). The SYBR-Green kit was purchased from Bio-Rad (Hercules, CA, USA).

Animals. Adult male Sprague-Dawley (SD) rats were purchased from the Medical Experimental Animal Center of Guangdong Province (Guangzhou, Guangdong, China). Rats were provided with standard rodent chow and water ad libitum under a natural day/night cycle. All the experimental protocols were approved by the Animal Ethics Committee of the First Affiliated Hospital of Shenzhen University (Shenzhen, Guangdong, China).

Experimental protocol. In total, $18 \mathrm{SD}$ rats were randomly divided into three groups, with each group containing six animals. Animals in group 1 underwent sham surgery (sham group). Animals in group 2 underwent bile duct ligation (BDL) $24 \mathrm{~h}$ subsequent to physiological saline pretreatment (control group), whilst animals in group 3 underwent BDL $24 \mathrm{~h}$ subsequent to histone pretreatment (HPC group).

Prior to surgery, rats were fasted for $12 \mathrm{~h}$ with water ad libitum. Each rat was weighed and anesthetized with $10 \%$ chloral hydrate $(300 \mathrm{mg} / \mathrm{kg})$ intraperitoneally. Following a midline incision, the common bile duct was exposed by careful separation from its surrounding soft tissue and a double-ligature with 5-0 silk suture was performed, and the bile duct was sectioned between the ligatures. A two-layer running suture was then used for abdominal closure with 4-0 dexon and 2-0 nylon. The sham animals underwent the same surgical procedure with the exception of ligation and section of the common bile duct. Animals in the control and HPC groups were intraperitoneally administered $1 \mathrm{ml}$ physiological saline and $200 \mu \mathrm{g} / \mathrm{kg}$ histones from calf thymus, respectively, $24 \mathrm{~h}$ prior to BDL. All animals were euthanized 14 days subsequent to BDL with an overdose of chloral hydrate.

Sample collection. A second laparotomy was performed once the animals were anesthetized, 14 days after BDL. Following collection of the blood samples from the inferior vena cava, the liver was carefully dissected from its attachment and totally excised. The blood samples were stored at $4^{\circ} \mathrm{C}$ for biochemical analysis of total bilirubin (TB), direct bilirubin (DB) and alanine aminotransferase (ALT) levels in the serum. The left lobe of the liver was excised and flushed with physiological saline and then cut into two sections. One section was immediately frozen in liquid nitrogen and stored at $-80^{\circ} \mathrm{C}$ for the measurement of mRNA levels of TLR-4, TLR-9 and interleukin-6 (IL-6), whilst the other section was fixed in $40 \mathrm{~g} / 1$ paraformaldehyde for histopathological analysis.

Histopathological observations. Liver tissues from all the experimental animals were fixed in $40 \mathrm{~g} / 1$ formaldehyde and embedded in paraffin. For histopathological evaluation, 4-mm slides were stained with hematoxylin and eosin. The sections were scored by an experienced hepatopathologist in a blinded manner. The histological activity index (HAI) scoring system has been previously used to evaluate histopathology in BDL rats $(18,19)$. In the present study, a modified HAI scoring system was used, which included the following lesions: piecemeal necrosis, confluent necrosis, focal (spotty) lytic necrosis, apoptosis, focal inflammation and portal inflammation.

The levels of bile duct proliferation were also scored by an experienced hepatopathologist in a blinded manner on a scale between 0 and 2, with 0 denoting absent or mild; 1 moderate and 2 severe proliferation (20). Neutrophils that accumulated in the liver were counted in a blinded manner in 20 randomly selected fields using a microscope (Olympus BX50-32H01; Olympus, Tokyo, Japan; magnification, $x 400)$. The data are expressed as the number of polymorphonuclear neutrophils per high-power field (PMNs/HPF).

Blood biochemistry. The results from the histopathological analysis were verified biochemically by measuring the serum levels of ALT, TB and DB in each experimental group using an autoanalyzer (Hitachi 7600-020; Hitachi, Tokyo, Japan).

Quantitative polymerase chain reaction ( $q P C R)$. Rat liver samples $(0.1 \mathrm{~g} /$ per sample $)$ stored at $-80^{\circ} \mathrm{C}$ were homogenized in $1 \mathrm{ml}$ TRIzol reagent and the total RNA was isolated in accordance with the manufacturer's instructions. The synthesis of cDNA was performed using the RevertAid First Strand cDNA Synthesis kit. The house-keeping gene $\beta$-actin was used as an internal control to analyze the mRNA expression levels of TLR-4, TLR-9 and IL-6. The sequences of the PCR primers were designed based on cDNA sequences from GenBank (http://www.ncbi.nlm.nih.gov/genbank/), and were as follows: TLR-4, forward 5'-CGCTCTGGCATCATCTTCAT-3' and reverse 5'-CTCCTCAGGTCAAAGTTGTTGC3'; TLR-9, forward 5'-TGAGCTACAACAGCCAGCCA-3' and reverse 5'-AATGTCATTGTGTGCCAGGC-3'; IL-6, forward 5'-GTCAACTCCATCTGCCCTTCAG-3' and reverse 5'-GGTCTGTTGTGGGTGGTATCCT-3'.

qPCR was performed using SYBR-Green PCR master mix according to the manufacturer's instructions and each sample was analyzed in duplicate. The mRNA levels of each of the genes being investigated were quantified using the ABI 7700 Sequence Detection System (Applied Biosystems, Warrington, UK) using the comparative methods. The quantity of mRNA was calculated using the $\Delta \Delta \mathrm{Ct}$ method. $\mathrm{Ct}$ values for each gene were normalized to the $\mathrm{Ct}$ value of $\beta$-actin $\left(\Delta \mathrm{Ct}=\mathrm{Ct}_{-}{ }_{\text {-actin }}-\mathrm{Ct}_{-}{ }_{\text {target }}\right)$. The results are presented as mRNA fold change: $2^{-\Delta \Delta \mathrm{Ct}}\left(\Delta \Delta \mathrm{Ct}=\Delta \mathrm{Ct}_{\text {-sham }}-\Delta \mathrm{Ct}_{\text {-HPC }}\right.$, in the HPC group or $\Delta \Delta \mathrm{Ct}=\Delta \mathrm{Ct}_{\text {-sham }}-\Delta \mathrm{Ct}_{\text {-control }}$, in the control group).

Statistical analysis. One-way analysis of variance, with subsequent post-hoc least significant difference tests and Bonferroni tests, was used for comparison between the experimental groups with continuous variables. Differences in the distribution of histopathological scores (modified HAI scores, bile duct proliferation scores and PMNs/HPF) between the groups were assessed using the Mann-Whitney U test. $\mathrm{P}<0.05$ was considered to indicate a statistically significant difference. 
All analyses were performed using SPSS statistical software version 10.0 (SPSS, Inc., Chicago, IL, USA).

\section{Results}

Macroscopic observations. Animals that underwent sham surgery (sham group) showed no alterations in the clinical conditions, specifically in normal activity, no irritability, no vertical hair, normal body weight, no yellowed tails, no darkened urine and no pale feces. In the control and HPC groups however, $24 \mathrm{~h}$ after surgery the clinical conditions of the animals deteriorated, as shown by decreased activity, irritability, vertical hair, body weight loss, yellowed tails, darkened urine and pale feces. All the animals survived until the end of the experiment. Jaundice was observed in the visceral and parietal peritoneum of all animals with the exception of animals in the sham group. Varying degrees of ascites, enlarged livers and dilated bile ducts above the obstruction point were also observed in all animals with the exception of those in the sham group.

Microscopic observations. No histological alterations were observed in animals in the sham group. Following euthanasia on day 14 after BDL, severe liver injury was observed in the control group, as indicated by an increase in neutrophil infiltration into the liver tissue, as well as an increase in ductal proliferation and significantly higher modified HAI scores $(\mathrm{P}<0.05)$ compared with the sham group. (Fig. 1; Table I).

Histone preconditioning significantly ameliorated the OJ liver injury induced by BDL in the control group, as indicated by a significant reduction in neutrophil infiltration into the liver tissue and decreased modified HAI scores of animals in the HPC group compared with the control group $(\mathrm{P}<0.05$; Fig. 1; Table I). The results of the histopathological analysis, including the PMNs/HPF, the bile duct proliferation scores and the modified HAI scores, of the three groups are summarized in Table 1. No significant difference was identified in the ductal proliferation scores between the HPC group and the control group $(\mathrm{P}>0.05)$.

Blood biochemistry results. BDL in the control group resulted in significantly elevated serum levels of TB and DB (Fig. 2) compared with the sham group, which suggests that the experimental OJ model was successfully induced. The serum levels of $\mathrm{TB}$ and $\mathrm{DB}$ in rats preconditioned with histone proteins prior to being subjected to BDL were not significantly different from those in the control animals $(\mathrm{P}>0.05)$, indicating that the degree of cholestasis was similar in the two experimental groups (Fig. 2). OJ liver injury induced by BDL was prominent in the control group, as shown by the significantly increased serum levels of ALT (Fig. 3) compared with the sham group $(\mathrm{P}<0.05)$. In accordance with the results of histopathological studies, histone preconditioning significantly ameliorated OJ-induced liver injury induced by BDL. The serum levels of ALT were significantly lower than that of the control group ( $\mathrm{P}<0.05$; Fig. 3).

mRNA expression of IL-6, TLR-4 and TLR-9. Using qPCR, it was demonstrated that the mRNA expression levels of IL-6 were significantly upregulated by BDL in the control group compared with the sham group $(\mathrm{P}<0.05$; Fig. 4$)$, which is consistent with previous studies (21). However, compared with the control group, histone preconditioning (HPC group) significantly downregulated the mRNA expression levels of IL-6 ( $\mathrm{P}<0.05$; Fig. 4). In addition, BDL in the control group significantly upregulated the mRNA expression levels of TLR-4 and TLR-9 ( $\mathrm{P}<0.05$; Fig. 5) compared with the sham group, which is consistent with previous studies (4). In the present study it was demonstrated that histone preconditioning significantly ameliorated the upregulation of the mRNA expression levels of TLR-4 and TLR-9. Animals preconditioned with histones expressed significantly lower mRNA levels of TLR-4 and TLR-9 ( $\mathrm{P}<0.05$; Fig. 5) compared with animals in the control group.

\section{Discussion}

$\mathrm{OJ}$ is a common clinical condition, which has been extensively studied, and is capable of inducing severe liver injury (2). Surgical, endoscopic and interventional decompressions are the primary treatment strategies for patients with OJ. However, biliary intervention has been demonstrated to augment inflammatory cell infiltration and aggravate OJ-induced liver injury $(22,23)$. Preconditioning with DAMP molecules has been demonstrated to be effective in protecting organs from injury in stressed situations (12-15). Preconditioning with hyperthermia $\left(<42^{\circ} \mathrm{C}\right.$ for $\left.20 \mathrm{~min}\right) 12 \mathrm{~h}$ prior to being subjected to BDL was found to significantly ameliorate OJ-induced liver injury $(9,10)$. However, hyperthermia is difficult to achieve in the clinic, thus, an effective pharmaceutical drug would be the preferred option.

Histones are a newly identified DAMP molecule, however, the preconditioning effect of histones on OJ-induced liver injury remains to be elucidated. In the present study, the effect of histone preconditioning on OJ-induced liver injury in rats was investigated. Preconditioning with HMGB1 (20 $\mu \mathrm{g} /$ mouse) has been demonstrated to significantly protect against hepatic I/R injury, and preconditioning with LPS $(100 \mu \mathrm{g} / \mathrm{kg}$ ) has been demonstrated to significantly protect against hepatic I/R injury, so in our study we preconditioned experimental animals with $200 \mu \mathrm{g} / \mathrm{kg}$ histone proteins $(16,24)$. The present study demonstrated that liver injury in animals in the HPC group was significantly ameliorated by histone preconditioning compared with the control group, as indicated by significant differences in the degree of necroinflammation and a decrease in the number of neutrophils infiltrating into the liver tissue. The serum levels of ALT were significantly lower in the HPC group compared with the control group, indicating that necrosis of hepatocytes was prevented, which is consistent with the results from the histopathological analysis.

Inflammation has been revealed to be important in the development of OJ-induced liver injury (2). In addition, the proinflammatory cytokine IL-6 has been demonstrated to be important in the pathogenesis of OJ-induced liver injury (21). Endotoxemia has been found to occur in patients with jaundice and experimental OJ animals, and LPS was demonstrated to be capable of inducing the release of proinflammatory cytokines, including TNF- $\alpha$ and IL-6 $(25,26)$. Therefore, in the present study, the expression levels of IL-6 in each group were investigated. Using qPCR, it was demonstrated that the mRNA 
Table I. Histopathological score of ductal proliferation, modified HAI and the number of PMNs/HPF in the three groups.

\begin{tabular}{lccr}
\hline & Sham & HPC & Control \\
\hline Ductal proliferation & 0 & 1 & $2.0^{\mathrm{a}}$ \\
PMNs/HPF & 0 & 2 & $7.5^{\text {a,b }}$ \\
HAI & 1.5 & 5 & $9.5^{\text {a,b }}$ \\
\hline
\end{tabular}

OJ liver injury induced by BDL was prominent in the control group, shown by the increase in neutrophil infiltration into the liver tissue, as well as an increase in ductal proliferation and significantly higher modified HAI scores. Histone preconditioning significantly ameliorated the OJ liver injury induced by BDL, as indicated by the significant decrease in neutrophil infiltration into the liver tissue and lower modified HAI scores. ${ }^{a} \mathrm{P}<0.05$, compared with the sham group; ${ }^{b} \mathrm{P}<0.05$, compared with the HPC group. OJ, obstructive jaundice; BDL, bile duct ligation; HAI, histological activity index; PMNs/HPF, polymorphonuclear neutrophils per high-power field.
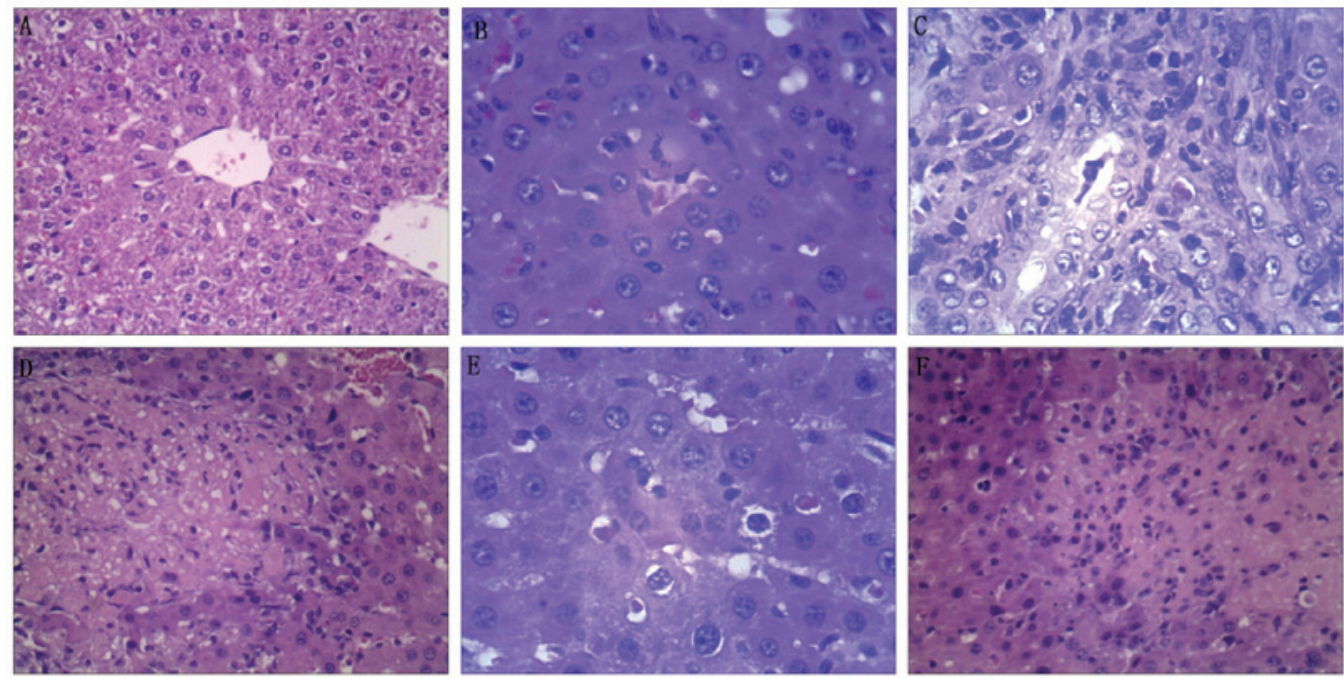

Figure 1. Effects of histone preconditioning on obstructive jaundice-induced liver injury indicated by hematoxylin and eosin staining. (A) In sham rats no histological alterations were observed (sham group). (B) Bile duct ligation in the control group resulted in severe liver injury, demonstrated by an increase in neutrophil infiltration into the liver, as well as an increase in (C) ductal proliferation and (D) severe necroinflammation. Histone preconditioning significantly ameliorated liver injury, shown by the (E) reduction in neutrophil infiltration into the liver and (F) and a decrease in the severity of necroinflammation.
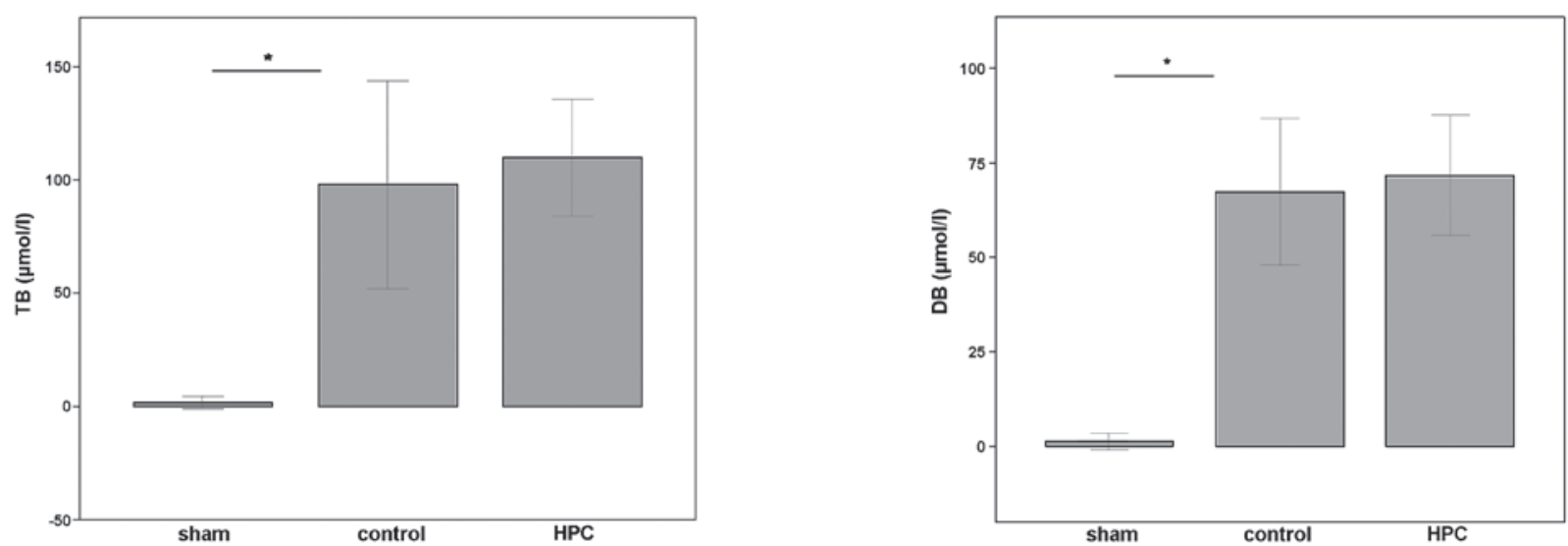

Figure 2. Alterations in serum TB and DB 14 days after BDL and the effect of histone preconditioning. Serum levels of TB and DB in the control group were significantly higher compared with the sham group 14 days subsequent to BDL. Histone preconditioning (HPC group) did not significantly alter the serum levels of TB and DB. "P<0.05, compared with the control using Student's t-test. TB, total bilirubin; DB, direct bilirubin; BDL, bile duct ligation.

expression levels of IL-6 in the control group were significantly higher compared with the sham group, whilst histone preconditioning significantly downregulated the mRNA expression of IL-6. The present study demonstrated that histone precon- ditioning protects against OJ-induced liver injury in rats by inhibiting the release of inflammatory mediators.

The preconditioning effects of HMGB1 and LPS on hepatic I/R injury were previously reported to be dependent 


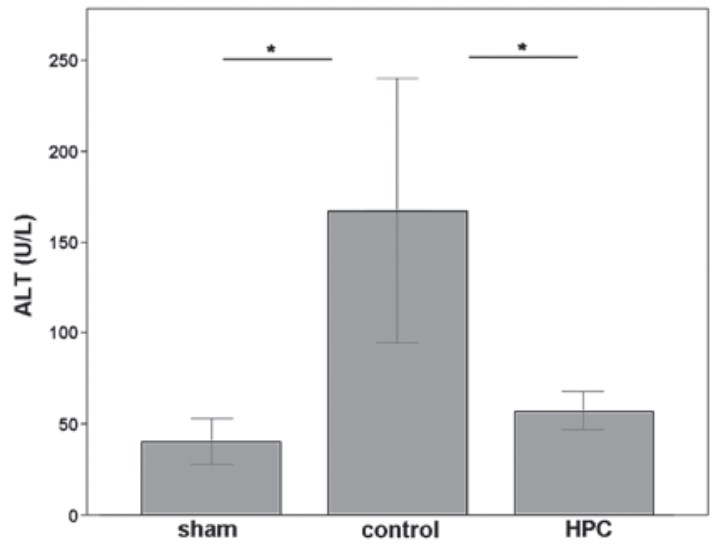

Figure 3. Effect of histone preconditioning on OJ-induced liver injury indicated by blood biochemistry analysis. OJ liver injury induced by BDL was marked in the control group, demonstrated by the significantly increased serum levels of ALT compared with the sham group. Histone preconditioning significantly ameliorated the OJ liver injury induced by BDL. ${ }^{*} \mathrm{P}<0.05$, compared with the control group. OJ, obstructive jaundice; BDL, bile duct ligation; ALT, alanine aminotransferase.

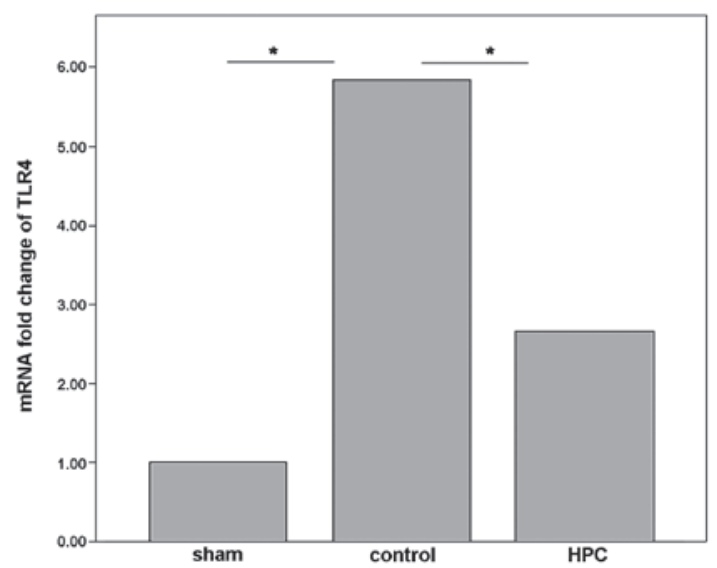

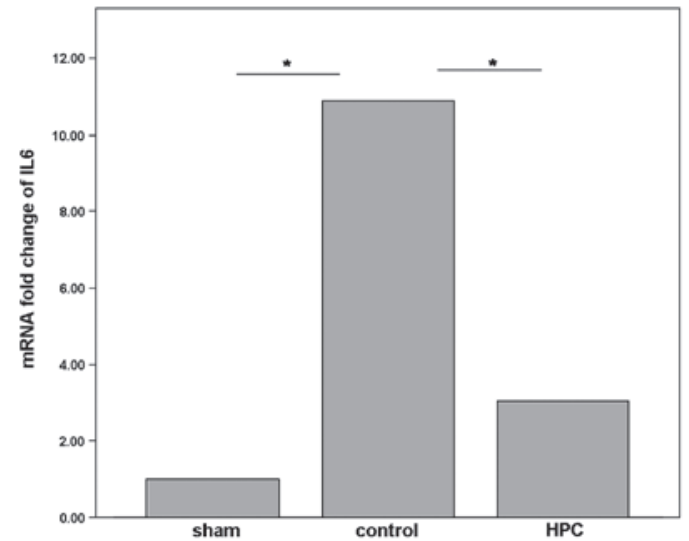

Figure 4. mRNA expression of IL-6 in the liver tissue. BDL in the control group significantly upregulated the mRNA expression of proinflammatory cytokine IL-6 in the liver tissue. Histone preconditioning (HPC group) significantly ameliorated OJ liver injury induced by BDL, as indicated by the decreased mRNA expression levels of IL- 6 in the liver tissue. ${ }^{\text {}} \mathrm{P}<0.05$, compared with the control group. IL-6, interleukin-6; BDL, bile duct ligation; OJ, obstructive jaundice.

Figure 5. mRNA expression levels of TLR-4 and TLR-9 in the liver tissue. Bile duct ligation in the control group significantly upregulated the mRNA expression levels of TLR-4 and TLR-9 in the liver tissue, while histone preconditioning (HPC group) significantly decreased the mRNA expression levels of TLR-4 and TLR-9 in the liver tissue. ${ }^{*} \mathrm{P}<0.05$, compared with the control group. TLR, Toll-like receptor.

on TLR4 expression (the receptor for HMGB1 and LPS). The receptors for histones have been demonstrated to be TLR-4 and TLR-9, therefore, the present study investigated the role of TLR-4 and TLR-9 in the process of histone preconditioning on OJ-induced liver injury $(16,17,24,27)$. The results from the qPCR analysis revealed that the mRNA expression levels of TLR4 and TLR9 in the liver tissue were significantly lower compared with the control group, which indicated that TLR4 and TLR9 may be involved in the process of histone preconditioning in OJ-induced liver injury.

Histone proteins are assembled with DNA to form nucleosomes in the nucleus and histone modifications have been demonstrated to be important in gene transcription $(28,29)$. Recently, histone proteins have been identified as DAMP molecules $(17,27)$. Furthermore, the present study demonstrated that histone proteins may be used as a preconditioning agent to protect against OJ-induced liver injury. Preconditioning may be achieved with a variety of stress responses and the most widely studied method is ischemia (30). The adaptive responses to preconditioning may enhance the body's tolerance to further stresses. Although the exact mechanism of preconditioning has not yet been fully elucidated, the activation of potassium channels, metabolic alterations and the generation of nitric oxide have been suggested to be involved (30-33). Investigations into the mechanisms underlying the protective effects of preconditioning have led to the application of DAMP molecules as preconditioning agents to protect against organ injury. However, for inflammatory diseases, for example OJ-induced liver injury, the interactions between different DAMP molecules, the network of proinflammatory cytokines and downstream signaling molecules are complex. Therefore, further studies are required to elucidate the mechanisms and the safety of the preconditioning molecules, particularly as DAMP molecules are known to be toxic.

In conclusion, histone preconditioning protects against OJ-induced liver injury in rats, and TLR4 and TLR9 may be involved in this process. Histone preconditioning may be a novel and promising therapeutic strategy for the treatment of OJ-induced liver injury and possibly other diseases. However, its safety and underlying mechanisms have not been 
fully elucidated. Thus, further investigations are necessary to ascertain the safety and therapeutic mechanisms underlying the protective effects of histone preconditioning.

\section{Acknowledgements}

The authors would like to thank the Department of Laboratory of The First Affiliated Hospital of Shenzhen University for assisting in the analysis of the biochemical data. The present study was supported by the Science and Technology Project Grant of Guangdong, China (no. 2012B031800349).

\section{References}

1. Tsuyuguchi T, Takada T, Miyazaki M, et al: Stenting and interventional radiology for obstructive jaundice in patients with unresectable biliary tract carcinomas. J Hepatobiliary Pancreat Surg 15: 69-73, 2008.

2. Gujral JS, Farhood A, Bajt ML and Jaeschke H: Neutrophils aggravate acute liver injury during obstructive cholestasis in bile duct-ligated mice. Hepatology 38: 355-363, 2003.

3. Koeppel TA, Trauner M, Baas JC, et al: Extrahepatic biliary obstruction impairs microvascular perfusion and increases leukocyte adhesion in rat liver. Hepatology 26: 1085-1091, 1997.

4. Huang YH, Wang PW, Tiao MM, et al: Glucocorticoid modulates high-mobility group box 1 expression and Toll-like receptor activation in obstructive jaundice. J Surg Res 170: e47-e55, 2011.

5. Klune JR, Billiar TR and Tsung A: HMGB1 preconditioning: therapeutic application for a danger signal? J Leukoc Biol 83: 558-563, 2008

6. He W, Fong Y, Marano MA, Gershenwald JE, Yurt RW, Moldawer LL and Lowry SF: Tolerance to endotoxin prevents mortality in infected thermal injury: association with attenuated cytokine responses. J Infect Dis 165: 859-864, 1992.

7. Colletti LM, Remick DG and Campbell DA Jr: LPS pretreatment protects from hepatic ischemia/reperfusion. J Surg Res 57: 337-343, 1994.

8. Ackerman M, Reuter M, Flohé S, Bahrami S, Redl H and Schade FU: Cytokine synthesis in the liver of endotoxin-tolerant and normal rats during hemorrhagic shock. J Endotoxin Res 7: 105-112, 2001

9. Mao SM, Zhang BM, Guan XD, Li J, Jia YB and Pan HY: The effect of hyperthermia preconditioning on the liver function of obstructive jaundice rats. J Pract Med 23: 3037-3838, 2007.

10. Mao SM, Zhang BM, Guan XD, Li J, Pan HY and Jia YB: The effect of hyperthermia pretreatment on the subgroups of T lymphocytes in obstructive jaundiced rats. Shijie Huaren Xiaohua Zazhi 15: 3035-3037, 2007.

11. Güllüoğlu BM, Bekraki A, Cerikçioğlu N, Söyletir G and Aktan AO: Immunologic influences of hyperthermia in a rat model of obstructive jaundice. Dig Dis Sci 46: 2378-2384, 2001.

12. Hu X, Jiang H, Cui B, et al: Preconditioning with high mobility group box 1 protein protects against myocardial ischemia-reperfusion injury. Int J Cardiol 145: 111-112, 2010.

13. Ha T, Hua F, Liu X, et al: Lipopolysccharide-induced myocardial protection against ischeamia/reperfusion injury is mediated through a PI3K/Akt-dependent mechanism. Cardiovasc Res 78 546-553, 2008

14. Zacharowski K, Frank S and Otto M: Lipoteichoic acid induces delayed protection in the rat heart, a comparison with endotoxin. Arterioscler Thromb Vasc Biol 20: 1521-1528, 2000.
15. Aneja R, Odoms K, Dunsmore K, Shanley TP and Wong HR: Extracellular heat shock protein-70 induces endotoxin tolerance in THP-1 cells. J Immunol 177: 7184-7192, 2006.

16. Izuishi $\mathrm{K}$, Tsung A, Jeyabalan $\mathrm{G}$, et al: Cutting edge: high-mobility group box 1 preconditioning protects against liver ischemia-reperfusion injury. J Immunol 176: 7154-7158, 2006.

17. Huang H, Evankovich J, Yan W, et al: Endogenous histones function as alarmins in sterile inflammatory liver injury through toll-like receptor 9. Hepatology 54: 999-1008, 2011.

18. Raetsch C, Jia JD, Boigk G, Bauer M, Hahn EG, Riecken EO and Schuppan D: Pentoxifylline downregulates profibrogenic cytokines and procollagen I expression in rat secondary biliary fibrosis. Gut 50: 241-247, 2002.

19. Ishak K, Babtista A, Bianchi L, Callea F, De Groote J, Gudot F, Denk H, Desmet V, Korb G, MacSween RN, Phillips MJ, Portmann BG, Poulsen H, Scheuer PJ, Schmid M and Thaler H: Histological grading and staging of chronic hepatitis. J Hepatol 22: 696-699, 1995.

20. Hammel P, Couvelard A, O'Toole D, et al: Regression of liver fibrosis after biliary drainage in patients with chronic pancreatitis and stenosis of the common bile duct. N Engl J Med 344: 418-423, 2001.

21. Kimmings AN, van Deventer SJ, Obertop H, Rauws EA, Huibregtse $\mathrm{K}$ and Gouma DJ: Endotoxin, cytokines, and endotoxin binding proteins in obstructive jaundice and after preoperative biliary drainage. Gut 46: 725-731, 2000.

22. Chuang JH, Chang NK, Huang CC, et al: Biliary intervention augments chemotactic reaction and aggravates cholestatic liver injury in rats. J Surg Res 120: 210-218, 2004.

23. Martignoni ME, Wagner M, Krähenbühl L, et al: Effect of preoperative biliary drainage on surgical outcome after pancreaticoduodenectomy. Am J Surg 181: 52-59, 2001

24. Sano T, Izuishi K, Hossain MA, Kakinoki K, Okano K, Masaki T and Suzuki Y: Protective effect of lipopolysaccharide preconditioning in hepatic ischaemia reperfusion injury. HPB (Oxford) 12: 538-545, 2010.

25. Bemelmans MH, Gouma DJ, Greve JW and Buurman WA: Cytokines tumor necrosis factor and interleukin-6 in experimental biliary obstruction in mice. Hepatology 15: 1132-1136, 1992.

26. Van Bossuyt H, Desmaretz C, Gaeta GB and Wisse E: The role of bile acids in the development of endotoxemia during obstructive jaundice in the rat. J Hepatol 10: 274-279, 1990.

27. Xu J, Zhang X, Monestier M, Esmon NL and Esmon CT: Extracellular histones are mediators of death through TLR2 and TLR4 in mouse fatal liver injury. J Immunol 187: 2626-2631, 2011.

28. van Holde KE (ed): Histone modifications. In: Chromatin. Volume 4, 1st edition. Springer New York, NY, pp111-148, 1988.

29. Strahl BD and Allis CD: The language of covalent histone modifications. Nature 403: 41-45, 2000.

30. Opie LH and Sack MN: Metabolic plasticity and the promotion of cardiac protection in ischemia and ischemic preconditioning. J Mol Cell Cardiol 34: 1077-1089, 2002.

31. Chen W, Glasgow W, Murphy E and Steenbergen C: Lipooxygenase metabolism of arachidonic acid in ischemic preconditioning and PKC-induced protection in heart. Am J Physiol 276: H2094-H2101, 1999.

32. Schulz R, Rose J and Heusch G: Involvement of activation of AT dependent potassium channels in ischemic preconditioning in swine. Am J Physiol 267: H1341-H1352, 1994.

33. Marber MS, Latchman DS, Walker JM and Yellon DM: Cardiac stress protein elevation 24 hours after brief ischemia or heat stress is associated with resistance to myocardial infarction. Circulation 88: 1264-1272, 1993. 